\title{
SISTEMA JURÍDICO Y REGLA DE RECONOCIMIENTO
}

1.

C erbert Hart sostiene la tesis según la cual, la existencia de un sistema jurídico

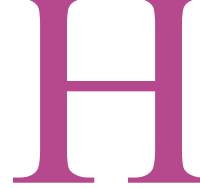
depende necesariamente de la utilización compartida de criterios de validez jurídica. Compartida al menos por los integrantes de la estructura del gobierno $\mathrm{y}$, en especial, por los individuos funcionalmente dedicados a la resolución de conflictos mediante normas jurídicas, esto es, por el conjunto de los jueces. «Existencia» significa aquí existencia empírica, y también es un dato fáctico aquel uso conjunto de criterios. Por otro lado, por «criterio de validez» se entiende, en primer lugar, criterio de identificación de normas jurídicas, a saber, precisamente de las normas que integran el sistema en cuestión. De hecho, según Hart, esto sucede porque, salvo problemas de patología del sistema -como denomina a las crisis jurídico-políticas- ${ }^{1}$, los funcionarios aceptan la misma regla de reconocimiento ${ }^{2}$. Justamente, con la introducción de la noción de «regla de reconocimiento» comienzan las dificultades para arribar a un acuerdo sobre el alcance de la propuesta de Hart. El contenido mínimo de la formulación lingüística de semejante regla es la mención de criterios de validez, o lo que es lo mismo -al menos, en principio- de criterios de identificación ${ }^{3}$, : una norma pertenece al sistema jurídico $\mathrm{S}, \mathrm{si}, \mathrm{y}$ sólo si, satisface alguno de los criterios formulados en la regla de reconocimiento de $\mathrm{S}$. La cuestión sobre la que puede versar uno de los desacuerdos -como el que mantienen Eugenio \footnotetext{
146 y sigs.

${ }^{2}$ Hart, op. cit., págs. 125 y sigs.

${ }^{3}$ «Decir que una determinada regla es válida es reconocer que ella satisface todos los requisitos establecidos en la regla de reconocimiento y, por lo tanto, que es una regla del sistema.» Hart, op. cit., pág. 129.
}

${ }^{1}$ H. L. A. Hart, El concepto de Derecho (traducción de G. Carrió). Buenos Aires, 1963, págs. 
Bulygin y Juan Ruiz 
Manero- ${ }^{4}$, consiste en preguntarse cuál es la actitud de los jueces, frente a una regla de reconocimiento. Existen dos respuestas posibles. Según la primera, los jueces «usan» la misma regla de reconocimiento, y esto es equivalente a afirmar que utilizan los mismos criterios de validez (identificación) de normas jurídicas, sencillamente, porque «usar» esa regla significa aquí emprender una práctica de identificación. La otra actitud consiste en la «obediencia» de los jueces, la que supone, entonces, que aquélla les impone al menos una obligación o un deber.

Los lúcidos argumentos de Ruiz Manero y E. Bulygin en favor de una u otra alternativa, muestran la pertinencia de tomar en serio, desde la perspectiva de una noción plausible de «sistema jurídico», un análisis ulterior de lo que se encuentra implicado en la adopción del «punto de vista interno» por parte de los jueces. Cuestión asociada a la idea según la cual, éstos obedecen la regla de reconocimiento hartiana. Me propongo, en lo que sigue, considerar algunas líneas posibles para la continuación de ese análisis. Por lo demás, hay que advertir que los términos «usar» y «obedecer» no pretenden reflejar la ambigua terminología de Hart, sino más bien, dar cuenta de la consideración de la regla de reconocimiento como «regla conceptual» o alternativamente, como «regla de conducta» 5 .

2. La concepción del derecho como sistema se encuentra asociada con el requerimiento -teórico, en principio- de contar con criterios para la identificación de normas jurídicas. Esta actividad presupone una noción general de «norma jurídica» y se dirige, en cambio, a la cuestión de saber cuáles son las que componen un determinado conjunto normativo al que habitualmente se denomina orden jurídico. El término más general «derecho» parece mencionar, así, una clase de esos conjuntos. Para esa tarea. la noción de «sistema» contribuye a proporcionar un método de decisión relativo a la pregunta de si una norma determinada $\mathrm{N}$ pertenece o no pertenece a un cierto conjunto. Ello,

${ }^{4}$ Cfr. J. Ruiz Manero, Jurisdicción y normas, Centro de Estudios Constitucionales, Madrid. 1991: E. Bulygin, «Algunas consideraciones sobre los sistemas jurídicos», en el presente número de Doxa.

${ }^{5}$ Para Hart, el término «obediencia» no describe adecuadamente la relación con las normas de aquellos que las «aceptan». También, los meros destinatarios las obedecen si ajustan su comportamiento a lo que aquellas prescriben. Además, «utilizar» menciona también, de manera general, a la actitud de los que adoptan el punto de vista interno frente a la regla de reconocimiento. Por ejemplo, Hart, op. cit., págs. 143 y, 125. Un análisis de las relaciones posibles entre normaslconductas se encuentra en P. Navarro, La eficacia del Derecho, Centro de Estudios Constitucionales, Madrid, 1990. 
porque un sistema se compone del conjunto más una cierta relación entre sus elementos, de manera que esa cuestión puede ser resuelta mostrando que $\mathrm{N}$ satisface la relación definitoria del sistema con otra norma N1, cuya pertenencia ha sido previamente establecida. La conclusión de Hart según la cual no puede hablarse de «sistema jurídico» sin las reglas secundarias parece adecuarse bien a esta concepción, porque son esas reglas las que permiten establecer relaciones entre normas.

Ahora bien, en una determinada localización de espacio y tiempo, la identidad de un definido sistema jurídico depende de criterios de la identificación de dos tipos de normas que se pueden denominar «normas independientes» $\mathrm{y}$ «normas dependientes» ${ }^{6}$. Las normas independientes son aquellas cuya pertenencia al sistema no depende de su relación con otras normas, y cumplen un papel semejante a los enunciados primitivos de un sistema teórico. A la inversa, la pertenencia de una norma dependiente se determina por su relación con otras normas del sistema. Hart, y también Ruiz Manero, usan la expresión «regla última», a la que identifican con la regla de reconocimiento, que parece corresponder con la noción de norma independiente. No obstante, esa terminología hartiana tiene un fuerte compromiso con la justificación de normas y conductas porque está asociada con la adopción del punto de vista interno. La noción de «norma independiente» es, en cambio, neutral en la medida en que es un artificio utilizable sin más, en el procedimiento teórico de identificación de normas, sin abrir juicio con respecto a su función en la actividad justificatoria de los destinatarios de las normas.

Para definir un sistema $\mathrm{S}$ es preciso, entonces: a) Proporcionar un criterio de pertenencia de las normas independientes, el que puede consistir en su mera indicación extensional. b) Definir la relación $\mathrm{R}$ (o relaciones) que permita establecer la pertenencia de las normas dependientes. Este es el procedimiento que utiliza Bulygin para construir un modelo de orden jurídico. Sin embargo, los teóricos del derecho -entre ellos Hart- no se conforman con una enumeración extensional para identificar las normas independientes: requieren que las mismas tengan una cierta propiedad $\mathrm{P}$ como condición de pertenencia. No existe inconveniente para ese requerimiento, salvo que la afirmación según la cual la norma independiente $\mathrm{N}$ tiene la propiedad $\mathrm{P}$ no tiene que

${ }^{6}$ Cfr. R. Caracciolo, «Rechtsordnung. System und Voraussagen des Rechts», en E. Garzón Valdés E. Bulygin (edts.) Argentinische Rethsteorie und Rechtsphilosophie Heute, Berlín, 1987, y R. Caracciolo, Sistema jurídico. Problemas actuales, Madrid, 1988. 
implicar, por la definición de P, que otra norma dependiente N1 satisface la relación R con N. Esta exclusión se impone para evitar un círculo en la identificación de las normas.

3. Por supuesto, una definición de este tipo es metasistemática, se formula en un nivel de lenguaje distinto de aquel en el cual se expresan las normas y es utilizable por un observador que se coloque en el punto de vista externo. Por tanto, sirve para la identificación teórica de un cierto sistema jurídico. Como no podía ser de otra manera, existe acuerdo acerca de la posibilidad de semejante formulación conceptual ${ }^{7}$. Pero mientras Bulygin propone denominar «regla de reconocimiento» a los criterios metasistemáticos de pertenencia, con lo que la excluye del sistema jurídico, Ruiz Manero, más allá de la eventual cuestión lingüística, en el fondo irrelevante, sostiene que existen razones de peso para conservar la concepción de la regla de reconocimiento tal como fuera formulada por Hart. Esto es, como la regla última del sistema -como una norma genuina de conducta-, y, por tanto, como aquella que viene a cumplir la función de una norma independiente: las restantes normas pertenecen, entonces, al sistema por su relación con la regla de reconocimiento. Además, están de acuerdo que los jueces tienen, al menos en los sistemas jurídicos contemporáneos, el deber de resolver los conflictos jurídicos mediante la aplicación de sus normas. Pero, mientras para Bulygin, este deber no puede ser impuesto por la regla de reconocimiento, puesto que es una regla conceptual, para Ruiz Manero necesariamente tiene que resultar de la misma regla de reconocimiento.

Pienso que la discrepancia puede explicarse por la adopción de distintas perspectivas acerca del análisis de un sistema jurídico. No sólo los teóricos (o cualquier observador externo) identifican las normas de un sistema $\mathrm{S}$, sino también los usuarios de $\mathrm{S}$, los que adoptan el punto de vista interno porque aceptan sus normas, utilizan criterios de validez jurídica en su práctica de identificación. De manera que se pueden distinguir criterios externos y criterios internos de identificación. Aunque sean idénticos -lo que puede ser discutible- para los aceptantes de las normas, tienen una calidad adicional, son vinculantes, esto es, imponen obligaciones o deberes. Y este carácter normativo no puede resultar de una definición. Tiene que existir en el sistema, entonces, una norma que asocie los criterios con un deber ${ }^{8}$. Precisamente,

${ }^{7}$ Cfr. Ruiz Manero, op. cit., págs. 137-139.

${ }^{8}$ Cfr. Ruiz Manero, op. cit., págs. 140-142, 172 y sigs.; también, P. M. S. Hacker, «Hart's Philosophy of Law», en P. M. S. Hacker y J. Raz, Law, Morality and Society. Essays in Honour of H. L. A. Hart, Oxford, 1977, pág. 23. 
se trata de la regla de reconocimiento. Que ciertos criterios se mencionen en el contenido de una norma para delimitar la conducta obligatoria, por ejemplo, en la interpretación estándar, el deber de los jueces de aplicar las normas de S, no la convierte en una regla conceptual. Así, la regla de reconocimiento puede tener la forma «obligatorio aplicar las normas que cumplan con los criterios $\mathrm{C} 1, \mathrm{C} 2 \ldots \mathrm{Cn}$ ». Entonces, si meramente se identifica la «regla de reconocimiento» con los criterios $\mathrm{C} 1$. $\mathrm{C} 2 \ldots \mathrm{Cn}$, se pierde la dimensión normativa del uso interno de esos criterios.

Sin esa dimensión, no sería posible dar cuenta del hecho de que, desde el punto de vista de los aceptantes, las normas son asumidas como razones para la acción, esto es, para justificar conductas. Especialmente, esto vale para los jueces que tienen que justificar jurídicamente sus decisiones en las normas que pertenecen al sistema ${ }^{9}$. Por consiguiente, el tratamiento de los criterios de pertenencia, no puede ser independiente de la cuestión de los criterios de justificación. En principio, no existe inconveniente para recurrir a esta versión de la regla de reconocimiento, en la medida en que la mención de los criterios habilita, indirectamente, la identificación de las normas dependientes del sistema. Pero, como finalmente pretendo mostrar, crea un problema insoluble a la hora de identificar la específica regla de reconocimiento de un cierto sistema $\mathrm{S}$.

4. Previamente, bajo el supuesto según el cual los jueces obedecen la regla de reconocimiento, vale la pena elucidar el alcance de esta dimensión normativa. En primer lugar, el concepto de «validez» y también el de «criterio de validez» se modifican subrepticiamente al ingresar en esa dimensión. Desde el punto de vista interno, una norma «válida» N no es, simplemente, aquella que satisface un criterio de pertenencia, sino además, una norma que debe ser obedecida, si se trata de una norma primaria ${ }^{10}$. Entonces, un «criterio de validez», no es sólo un criterio de identificación, sino además, el fundamento de la validez

${ }^{9}$ Cfr, R. Caracciolo, «Justificación normativa y pertenencia. Modelos de decisión judicial», en Análisis Filosófico, año 1988, núm. 2, Buenos Aires. 1988.

${ }^{10}$ Cfr. E. Bulygin, «Norms, Normative Propositions and Legal Statements», en Contemporany Philosophy. A New Survey, The Hague Boston London, 1982. Según MacCormick, la regla de reconocimiento no determina la validez o la existencia de las normas a las que se refiere. sino lo que cuenta como fundamentos válidos para las decisiones judiciales. Claramente, se tratan de usos distintos de «validez», en el segundo equivale a «obligatoriedad», N. MacCormick, H. L. A. Hart, Londres, 1981, págs, 114-115. 
de $\mathrm{N}$, esto es, la razón por la cual $\mathrm{N}$ debe ser obedecida. Ello explica que Hart denomine también «criterio de validez» de N, a la norma N1 que autoriza a la autoridad X la promulgación de $\mathrm{N}^{11}$. Pero claramente, una norma de competencia no suministra el criterio conceptual que se precisa para saber si $\mathrm{N}$ pertenece al sistema, sin perjuicio que el respectivo criterio de identificación, como el propio Hart lo señala ${ }^{12}$, se refiera al hecho de la promulgación autorizada de $\mathrm{N}$ como una de las condiciones suficientes de pertenencia. No obstante, se trata de usos distintos de las expresiones «validez» y «criterio de validez». Por un lado, «validez» significa pertenencia a un sistema, y «criterio de validez» la definición de la relación de pertenencia, por el otro, significan obligatoriedad y fundamento de obligatoriedad respectivamente. Las normas «válidas» normativamente, no sólo imponen «deberes», en sentido débil, para sus destinatarios; además son «obligatorias», existen razones para justificar el deber ${ }^{13}$. A Bulygin le interesa el primer sentido de la noción de «validez», a Ruiz Manero, el segundo ${ }^{14}$.

5. No hay duda que si se admite la versión estándar de la regla de reconocimiento, ésta parece destinada a suministrar «criterios de validez» en ambos sentidos. No obstante, no es nada claro cuál es papel de la regla de reconocimiento entendida como fundamento normativo, esto es, como criterio de justificación. Cuestión que es equivalente a la de saber cuál es la clase de actos que la regla torna obligatorios y cuál la clase de sus destinatarios. En la discusión de este punto, tanto Bulygin como R. Manero acuerdan en limitarla a los actos de aplicación de las normas del sistema, lo que implica que, al menos, sus destinatarios

${ }^{11}$ Hart, El concepto de Derecho, op. cit., pág. 133.

12 «Obviamente, habrá una conexión muy estrecha entre las reglas de cambio las de reconocimiento: porque donde existan las primeras, las últimas necesariamente incorporaran una referencia a la legislación...», Hart, op. cit., pág. 119. También Raz advierte que para decidir una cuestión de «validez» en el sentido de pertenencia, lo que hace falta no es otra norma sino un criterio teórico jurídico de identificación. J. Raz, El concepto de sistema jurídico (traducción de R. Tamaw), México, 1986, pág. 238.

${ }^{13}$ Una discusión sobre la distinción, en este contexto, entre «deberes» en sentido débil y «obligaciones» o «deberes» en sentido fuerte, se encuentra en J. Raz: «Promises and Obligations», en P. M. S. Hacker J. Raz (ed.), Law, Morality and Society. Essays in Honour of H. L. A. Hart, op. cit.

${ }^{14}$ Ello resulta del siguiente párrafo de Ruiz Manero: «...Y eso es precisamente la regla de reconocimiento: una norma jurídica que establece cuáles son los criterios últimos de validez jurídica, esto es, una norma que establece, con carácter último, cuáles son las normas que los jueces tienen el deber de aplicar. Ruiz Manero, J., Normas, op. cit., pág. 142 (subrayados míos). 
son los jueces. Sin embargo, hay otras alternativas para determinar el contenido de la regla de reconocimiento. La imprecisión resulta aquí de la ambigüedad de la expresión «reconocimiento» en la teoría de Hart. En primer lugar, significa la operación de identificar una norma, la que tiene un manifiesto carácter cognoscitivo y presupone el uso de un criterio conceptual $^{15}$. Pero además, reconocer una norma es lo mismo que aceptarla, esto es, considerar su contenido como pauta de comportamiento correcto ${ }^{16}$. La aceptación es una actitud práctica y no teórica y supone la disposición a asumirla como una razón para la acción ${ }^{17}$. Como indica Nell MacCormick, la adopción del punto de vista interno comprende tanto la actitud cognoscitiva como la práctica. Lo que es consecuencia de la constatación obvia de que para aceptar una norma previamente hay que conocerla ${ }^{18}$. De manera que se tienen tres tipos de comportamiento que pueden constituir el contenido obligatorio de la regla de reconocimiento: identificación, aplicación y aceptación de las normas del sistema. El hecho de que la aceptación es, finalmente, una actitud, no la excluye como alternativa, toda vez que aquella se manifiesta externamente en reacciones críticas de aprobación o rechazo ${ }^{19}$.

A pesar de la tendencia, también imprecisa, de incorporar como contenido del deber impuesto por la regla de reconocimiento a los actos de identificación de normas, por cierto ello no basta para dar cuenta de la dimensión normativa. Ello porque, en todo caso, se trata de operaciones intelectuales que no implican compromiso alguno con las normas así «identificadas». «Reconocer» en este sentido, no conduce a la aceptación de las normas del sistema y sólo significa el uso de un criterio conceptual, que puede efectuarse desde el punto de vista externo. Inversamente, los que aceptan las normas, esto es, los que las «reconocen» en el segundo sentido, tienen también necesariamente que recurrir a un criterio de identificación, por ejemplo, al que indica a la legislación como una «fuente» normativa. De manera que la aceptación compartida de normas por parte de los jueces (y demás

${ }^{15}$ Cfr. Hart, El concepto de Derecho, op. cit., págs. 118-119.

${ }^{16}$ Cfr. Hart, El concepto de Derecho, op. cit., pág. 76. También, Hart usa «reconocimiento» en el sentido de «aceptación» en su ensayo «Commands and Authoritative Legal Reasons», ahora incluido en J. Raz (ed.), Authority, Oxford, 1990, por ejemplo, págs. 105-106.

${ }^{17}$ Cfr. Hart, «Commands and Authoritative Legal Reasons», cit. v R. Manero, Jurisdicción y Normas, op. cit., págs. 173 y sigs.

${ }^{18}$ N. MacCormick, H. L. A. Hart, op. cit., págs. 33 y sigs.

${ }^{19}$ Cfr. P. M. S. Hacker, «Hart's Philosophy of Law», cit. págs. 13 s, sigs. 
funcionarios) supone el uso compartido de criterios de identificación. Pero de ello no se sigue que, además, obedezcan una norma según la cual deben usar una regla conceptual. Semejante norma, desde el punto de vista de los aceptantes es insuficiente como estándar de justificación o, en el mejor de los casos, superflua.

6. La cuestión de saber si los actos de aplicación de las normas del sistema por parte de los jueces son buenos candidatos a constituir el contenido del deber impuesto por la regla de reconocimiento, merece un análisis más detallado. No obstante el acuerdo generalizado para admitir que tal es el caso, se tienen argumentos para dudar si de este modo se explica la dimensión normativa que se le quiere atribuir. De manera general, ello resulta del hecho de que, si bien tanto la aplicación como la aceptación de normas implican su previa identificación, la aplicación de una norma $\mathrm{N}$ no implica su aceptación e, inversamente, la aceptación de $\mathrm{N}$ no supone lógicamente su aplicación. Por consiguiente, se tiene que efectuar una elección entre las dos alternativas. Ello, porque hay que distinguir la clase de los «aceptantes» de las normas y la clase de sus «destinatarios». Aceptantes son los que consideran a $\mathrm{N}$ como pauta de comportamiento correcto, esto es, los que admiten su fuerza «obligatoria», destinatarios son aquéllos a los que el contenido de $\mathrm{N}$ impone un «deber»en sentido débil o concede una permisión o un facultamiento. Ambas clases no tienen por qué coincidir y habitualmente no coinciden ${ }^{20}$. En lo que sigue, usaré «deber» para mencionar, meramente, el contenido de una norma.

Hay que elucidar, también, lo que cabe entender aquí por «aplicación» de una norma por parte de los jueces. Un deber que imponga a los jueces la aplicación de las normas del sistema $\mathrm{S}$, está destinado a asegurar la necesidad funcional de resolver los conflictos de manera autoritaria. Ello significa que tienen que adoptar decisiones, es decir, emitir las normas individuales en que consisten las sentencias, relativas al cumplimiento o incumplimiento de esas normas -normalmente normas generales por parte de sus destinatarios. Los jueces aplican, entonces, las normas de $\mathrm{S}$ cuando emiten sentencias que constituyen consecuencias de sus respectivos contenidos. Los sistemas contemporáneos

${ }^{20} \mathrm{Cfr}$. H. Kliemt, Las instituciones morales (traducción de J. M. Seña, revisión de E. Garzón Valdés y R. Zimmerling), Barcelona, 1986, págs. 192 y sigs. Este trabajo ofrece un excelente análisis de lo que Kliemt denomina «lógica del comportamiento» vinculado a la aceptación de normas dentro del paradigma de Hart. 
incluyen ese deber mediante disposiciones específicas que imponen a los jueces la obligación de decidir y la de fundamentar la sentencia en el derecho vigente. Por tanto, de tales normas sus destinatarios directos son los funcionarios judiciales. Pero hay un sentido aceptable para la presente discusión en que puede decirse que son, adicionalmente, los destinatarios indirectos de las que tienen que aplicar en ejercicio de su competencia: son las que determinan el contenido de las sentencias. Es este sentido el que adoptan Bulygin y Ruiz Manero para acordar que un código penal le impone al juez el deber de condenar al autor de un homicidio ${ }^{21}$. Es aceptable porque es evidente que mediante el sólo recurso al deber general de aplicar las normas de $\mathrm{S}$, es imposible determinar el contenido de la sentencia que, eventualmente, el juez debe dictar.

Sin duda, es indiscutible que las normas que imponen el deber general integran la lista de las normas dependientes o, en la otra terminología, derivadas, de los sistemas jurídicos actuales. En este sentido, su pertenencia es contingente. Pero, sostiene Ruiz Manero, ellas son insuficientes para fundamentar en última instancia ese deber. Para ello, es preciso, como también insiste MacCormick, que constituya el contenido de la regla de reconocimiento ${ }^{22}$. Ello significa no sólo que es constitutivo de la definición de «juez», sino, además, si se adopta la teoría de Hart, que se trata de un deber necesario, toda vez que la norma que lo impone pertenece necesariamente a cualquier sistema jurídico. Precisamente, porque esto es lo que sucede, por definición, con la regla de reconocimiento. No es seguro que esta tesis reproduzca la concepción originaria de $\operatorname{Hart}^{23}$. Pero más allá de ello,

${ }^{21}$ R. Manero, Jurisdicción y Normas, cit. pág. 141, y, E. Bulygin, «Algunas consideraciones sobre los sistemas jurídicos», cit.

${ }^{22}$ «Si decimos que la regla de reconocimiento establece lo que es una «norma obligatoria», y si decimos, con Hart, que es una regla que determina los «deberes» de los jueces, entonces todo lo que podemos significar con ello, es que ella establece cuáles reglas son obligatorias para los jueces como bases de decisiones justificadas acerca de lo correcto y lo incorrecto». N. MacCormick, $H$. $L$. A. Hart, cit. pág. 115.

${ }^{23}$ Hart expresamente indica que lo decisivo para el status de «juez» son las reglas secundarias de adjudicación que confieren potestades, y parece pensar que el deber de juzgar es meramente contingente al afirmar que aquellas pueden ser reforzadas mediante normas que lo impongan. Hart, $E l$ concepto de Derecho, cit. pág. 120. En «Commands and Authoritative Legal Reasons», dice ahora que ese deber está vinculado al oficio de juez, pero no afirma que lo tenga que imponer la regla de reconocimiento, op. cit., pág. 104. De cualquier modo el deber es innecesario si lo que se pretende es definir lo que es una «sentencia válida», en el sentido de pertenencia al sistema, porque las «potestades 
pienso que no es suficiente para dar cuenta de la dimensión normativa de la justificación de las decisiones judiciales, ni para explicar la función que Hart pretende atribuir a la regla de reconocimiento.

7. Si se admite, por un lado, que las distinciones anteriores son correctas, y, por el otro, como sucede normalmente, que el sistema incluye una norma dependiente $\mathrm{N}$, que impone un deber general de aplicación, se tienen, entonces, las siguientes consecuencias con respecto a los deberes de los jueces: i) El juez X puede aceptar la norma $\mathrm{N}$ y la norma N1, que establece un deber específico, y no ser destinatario indirecto de $\mathrm{N} 1$, lo que significa que la conjunción de ambas normas no le imponen a X ningún acto de aplicación. Esto sucede cuando X carece de competencia para resolver controversias mediante la utilización de N1. Es más, con frecuencia, en este supuesto le está prohibido su aplicación. Tal es el caso si es un juez con competencia en lo civil, en relación a la cuestión de saber si alguien cometió o no un delito. Sin embargo, $\mathrm{X}$ puede estar dispuesto a admitir que su colega $\mathrm{Y}$, con competencia en lo penal, ha adoptado una decisión justificada desde el punto de vista del contenido de N1. ii) El juez Z puede rechazar, lo que implica no aceptar, las normas $\mathrm{N}$ y N1, $\mathrm{y}$, no obstante, ser un destinatario indirecto de N1 y ello quiere decir tiene el deber de aplicar N1. Además, puede ser que, de hecho, Z aplique N1 en todos los supuestos en que debe hacerlo. No obstante, las normas $\mathrm{N}$ y N1 no constituyen aquí las razones subjetivas del comportamiento de Z. La situación es, por cierto, lógicamente posible ${ }^{24}$, y no puede descartarse acudiendo a la constatación empírica de la aceptación generalizada de las normas por parte de los jueces. Esa aceptación, condición necesaria para ingresar al punto de vista interno, indica que comparten los mismos criterios de justificación y que las aplican no por razones meramente subjetivas, sino porque las consideran vinculantes de manera compartida. Pero, precisamente, se trata de saber si el hecho de esa aceptación presupone, para su explicación, el cumplimiento por parte de los jueces de una norma ulterior que les impone un deber general de aplicación.

8. Supongamos que además de $\mathrm{N}$, es decir la norma dependiente que establece ese deber, la regla de reconocimiento, la norma independiente que tiene que servir en última instancia para justificar los actos de los jueces, tiene el mismo contenido

jurisdiccionales», esto es, las normas de competencia suministran suficiente criterio para ello.

${ }^{24}$ Cfr. P. M. S. Hacker, «Hart’s Philosophy of Law», cit. págs. 24-25. 
que N. Se tienen dos supuestos para analizar. En primer lugar, cuando los jueces son destinatarios indirectos con respecto a una norma $\mathrm{N} 1$, lo que quiere decir que tienen que emitir una sentencia de acuerdo al contenido de N1, la regla de reconocimiento es redundante, esto es, no es necesaria para establecer lo que se debe hacer. Si de la conjunción de $\mathrm{N}$ y N1 resulta que se tiene el deber de realizar el acto p, para cumplir con N y N1 hay que realizar p. Y si de la regla de reconocimiento resulta que se debe aplicar $\mathrm{N}$ y $\mathrm{N} 1$ -porque son normas del sistema- para cumplir con la regla de reconocimiento también hay que realizar el acto p. Pero no sólo es redundante para convertir a los jueces en destinatarios de un deber de aplicación. También es redundante para justificar, desde el punto de vista interno, el acto del juez. Para ello, es suficiente con aceptar las normas N y N1. No es preciso, como parece suponer Ruiz Manero, una serie de normas jerárquicamente escalonadas cada una de las cuales obliga a los jueces a realizar, finalmente, el mismo acto: $\mathrm{N}$ obliga a realizar el acto $\mathrm{p}, \mathrm{N} 1$ a cumplir $\mathrm{N}, \mathrm{N} 2$ a cumplir $\mathrm{N} 1, \ldots \mathrm{Nn}$ a cumplir $\mathrm{Nn}-1$. Todas, menos una, son redundantes con respecto al acto $\mathrm{p}$.

Una cuestión distinta es preguntarse por la justificación no ya de los actos impuestos por las normas, sino de las mismas normas. Entonces, se puede decir que se acepta $\mathrm{N}$ porque se acepta la autoridad que la emitió o porque se acepta la constitución, o por razones morales o prudenciales. Esta actividad es, por supuesto, relevante desde el punto de vista práctico y cuando para ello se recurre a otras normas (morales o jurídicas), a la inversa de lo que afirma Bulygin, es notorio que las normas se «usan»y no, simplemente, se «mencionan». Pero las normas «justificatorias» no pueden tener el mismo contenido que las que se pretende justificar. En tal caso no se tienen, en verdad, normas diferentes y cualquiera sea la jerarquía de las que están en cuestión, en tanto actitud práctica, esto es, a efectos de justificar un comportamiento la aceptación de una o de otra, son equivalentes.

En vista de ello, el argumento disponible para los que sostienen que el deber de aplicación se incluye en la regla de reconocimiento, consiste en mantener que, al menos, integra todos los sistemas jurídicos a los que no pertenece una norma dependiente $\mathrm{N}$ que imponga el deber de aplicación. Por cierto, esto es suficiente para convertir tal deber en un ingrediente necesario de cualquier sistema: o bien lo establece una norma dependiente o bien una norma independiente. Pero entonces, la que es contingente es la propia regla de reconocimiento.

Adicionalmente, en segundo lugar, si lo que se pretende es 
dar cuenta de aquella aceptación generalizada mediante el supuesto de una norma que los jueces obedecen y aceptan, un deber general de aplicación es insuficiente de manera notoria para explicar el caso de los jueces que aceptan las normas del sistema de las que no son sus destinatarios indirectos. No se puede decir aquí que las aceptan porque tienen un deber general de aplicarlas. El sentido común indica que no tienen ningún deber con respecto a la aplicación de esas normas, y, por consiguiente, no existen actos de aplicación que tengan que ser justificados. En cambio, se tienen los actos de aceptación de las normas, o más bien las manifestaciones de crítica y rechazo que los acompañan. También en este supuesto, para que esas normas constituyan razones para la acción, es suficiente que los jueces las acepten como criterios aptos para justificar comportamientos. No obstante si, de nuevo, el objetivo es explicar la aceptación mediante el expediente de afirmar una regla última que aquéllos siguen, esta regla tiene que constituir el criterio mediante el cual se justifican los actos de aceptación de las normas del sistema.

9. Se puede concluir, en consecuencia, que la hipótesis según la cual, para dar cuenta del uso de la regla de reconocimiento y sus funciones desde el punto de vista interno, es menester que ella imponga un deber general de aceptación, es enteramente razonable. Como puede darse el caso límite en que ninguno de los jueces acepte las normas de las que son destinatarios, lo que se precisa no es una norma que los convierta en destinatarios de todas las normas del sistema, sino una que los convierta en aceptantes comunes de esas mismas normas. Precisamente, ésta es la formulación que, con frecuencia, los intérpretes de Hart, incluyendo a Ruiz Manero, le otorgan a la regla de reconocimiento ${ }^{25}$. Pero una cosa son los actos de aplicación y otra los actos de aceptación. Y, por cierto, concuerda con la idea de que la aceptación de una norma básica constituye el punto de partida para participar en un «juego de lenguaje» en el que las normas no sólo son válidas porque satisfacen criterios de pertenencia, sino que son válidas porque son vinculantes, esto es, porque tienen fuerza obligatoria $^{26}$.

${ }^{25}$ «Aceptar la regla de reconocimiento viene a consistir, pues, en aceptar considerar los estándares jurídicos, con independencia de su contenido, como razones perentorias para la acción», Ruiz Manero, op. cit., 173. También es ilustrativa la cita de Raz que Ruiz Manero introduce en la pág. 179. Según Raz «Los jueces que aceptan la regla de reconocimiento aceptan una regla que les exige aceptar otras reglas...» (subrayados míos).

${ }^{26}$ H. Kliemt, Las instituciones morales, op. cit., págs. 188, 194 y sigs. 
No obstante, esta alternativa conduce también a un resultado paradójico. Como es sabido, la regla de reconocimiento es una regla social en el sentido de Hart. Existe, como cuestión de hecho, si, y sólo si, es eficaz. No se trata meramente de un cumplimiento acrítico el que se exige para esa existencia. Es necesario, además del cumplimiento, que sus destinatarios la acepten, es decir, la consideren pauta correcta de comportamiento. Habida cuenta que su contenido consiste en imponer un deber de aceptación, se la cumple si se aceptan las normas del sistema porque se acepta la regla de reconocimiento ${ }^{27}$. ¿Pero qué sucede si el sistema incluye una constitución escrita, cuya aceptación habilita la justificación de las normas inferiores? Si la constitución en cuestión no es aceptada generalmente por los jueces entonces la regla de reconocimiento correspondiente es ineficaz y, por tanto, no existe. Inversamente, si la constitución escrita es aceptada, ello significa que se tiene una práctica crítica y generalizada de obediencia o cumplimiento de sus disposiciones. Lo que incluye la aceptación por parte de los jueces de las normas promulgadas conforme a la constitución. Pero entonces, lo que existe es sólo una práctica crítica y resulta imposible discernir si ella corresponde a la aceptación de la constitución o a la aceptación de la regla de reconocimiento. En cualquier caso, como el propio Hart lo indica, es una duplicación innecesaria suponer que además de la constitución, existe otra norma al efecto que las disposiciones de la constitución deben ser obedecidas ${ }^{28}$. A fortiori ¿ello vale para una norma que imponga el deber de aceptar una constitución aceptada de hecho.

Lo que sucede es que una constitución aceptada de manera general es también una regla social. Como indica J. Coleman, se tienen dos nociones de «regla social». Conforme a la primera, que se corresponde con la idea de norma consuetudinaria, el comportamiento regular precede a la formulación de la regla, y determina su contenido. De acuerdo a la segunda, el contenido de la regla puede ser especificado con anterioridad a la existencia

${ }^{27}$ Se puede discutir también la teoría de Hart de la regla de reconocimiento preguntando si consigue dar cuenta de la normatividad del derecho. Después de todo, los comportamientos reiterados y las actitudes críticas no son nada más que hechos. Pero esa pregunta no es relevante para este trabajo. Conr. al respecto, L. Green, «Authority and Convention», en The Philosophical Quarterly, vol. 35, núm. 141, 1985.

${ }^{28}$ Hart, El concepto de Derecho, op. cit., pág. 310 (nota al capítulo VI). Se trata de una crítica a la teoría kelseniana de la norma básica, pero así formulada es directamente aplicable a la propia teoría de Hart. 
empírica de la práctica correspondiente ${ }^{29}$. Es el caso de una constitución eficaz. La cuestión de saber cuándo una constitución formalmente promulgada se convierte en «regla social» en este sentido, es idéntica a la de determinar en qué momento un hábito de conducta se transforma en una regla social $\mathrm{y}$, por consiguiente, no puede servir de objeción a la distinción.

De nuevo, el argumento para resolver la paradoja puede ser que, de todas formas, la regla de reconocimiento existe toda vez que un sistema carece de una constitución semejante. Es, por cierto, una salida aceptable, pero al costo de admitir, o bien que la regla de reconocimiento no es un componente necesario de cualquier sistema jurídico, o bien, que hay que identificarla con una constitución promulgada o consuetudinaria.

10. A esta altura, puede preguntarse por qué no retornar al punto de partida, que ahora aparece prematuramente desechado. Esto es, a los actos de identificación de normas, más allá del deber de aplicación y de aceptación. Después de todo, la tesis literal de Hart consiste en que cuando existe un sistema jurídico, al menos los jueces, identifican las normas con los mismos criterios de validez, donde «validez» ahora vuelve a tener el significado de «pertenencia». Este es un hecho notorio en los sistemas contemporáneos. Hasta aquellos jueces que no aceptan las normas -esto es, los que se colocan en el punto de vista externo tienen que admitir que la constitución, por ejemplo, pertenece al sistema S. Por tanto, más allá de los actos de aplicación o rechazo, es correcto prima facie decir que siguen una regla compartida de identificación, precisamente, la regla de reconocimiento. Como aceptar una regla conceptual no es lo mismo que aceptar las normas identificadas con los criterios que aquella establece, se puede admitir que impone a los jueces el uso obligatorio de esos criterios. Por cierto, se pierde aquí la función de la regla destinada a explicar la aceptación de las normas, y es difícil saber por qué habrían de considerar obligatorio los jueces esos criterios, sin aceptar, al mismo tiempo, las normas así identificadas.

No obstante, la regla de reconocimiento concebida de esta forma no puede integrar el mismo sistema de normas que las identificadas con los criterios mencionados en su contenido $^{30}$.

${ }^{29}$ Jules Coleman, «Negative and Positive Positivism», incluido en J. Coleman, Markets, Morals and the Law, Cambridge, 1988, pág. 344 (nota 12 de las págs. 9-20).

${ }^{30}$ A. Ruiz Miguel formula la incisiva propuesta de distinguir las nociones de «norma superior» $\mathrm{y}$ «regla última» a la que identifica con la regla de reconocimiento 
Un sistema $\mathrm{S}$, en un tiempo t y en un espacio e, se conforma con una norma (o normas) independiente y normas dependientes. Si una regla de reconocimiento pertenece a S entonces sólo puede ser una norma independiente. La pertenencia de las normas dependientes se determina por sus relaciones con esa regla. Por lo demás, en la teoría de Hart, un sistema se constituye sobre un cierto material normativo, a saber, verbigracia, las normas $\mathrm{N} 1, \mathrm{~N} 2 \ldots \mathrm{Nn}$, que son las efectivamente reconocidas por los jueces mediante el uso de los criterios, digamos, $\mathrm{C} 1, \mathrm{C} 2 \ldots \mathrm{Cn}$. Una condición de pertenencia impuesta, por definición, a la regla de reconocimiento consiste en que tiene que tener una cierta propiedad $\mathrm{P}$ : tiene que ser eficaz, tiene que ser utilizada por los jueces y ello significa que tiene que mencionar los criterios $\mathrm{C} 1, \mathrm{C} 2, \ldots \mathrm{Cn}$. De manera que: i) para conocer cuáles son las normas dependientes de S, hay que conocer cuáles son las que satisfacen los criterios $\mathrm{C} 1, \mathrm{C} 2, \ldots \mathrm{Cn}$ establecidos en la regla de reconocimiento, esto es, en la norma independiente de $\mathrm{S}$; ii) para conocer cuál es la regla de reconocimiento de $\mathrm{S}$, hay que saber cuáles son los criterios que son satisfechos por las normas dependientes de $\mathrm{S}$, es decir, por las normas N1, N2... Nn. Esto es así, porque un criterio de pertenencia $\mathrm{C}$ integra el contenido de la regla propuesta por Hart, si, y sólo si, existe al menos una norma aceptada $\mathrm{N}$ que satisface el criterio $\mathrm{C}$. Pero en la medida en que conduce a un procedimiento circular, semejante «sistema» no puede constituir un instrumento de identificación, ni para los que se colocan en el punto de vista externo, ni para los que adoptan el punto de vista interno. Esta constatación muestra, finalmente, que los criterios de pertenencia a un sistema -que después de todo, no es nada más que una construcción conceptual- no pueden integrar el sistema. Constatación, por lo demás, independiente de la cuestión de saber si los jueces «usan» u «obedecen» la regla de reconocimiento,

Barcelona, mayo de 1991

cimiento de un sistema, mientras, verbigracia, la constitución vendría a desempeñar el papel de «superior» o «suprema». Ello para resolver una antinomia asociada con el principio de jerarquía normativa. Se trata del primer paso que hay que dar para arribar a la conclusión según la cual la regla «última», es decir, la que suministra los criterios de validez, no puede pertenecer al mismo sistema de las normas que son «superiores» conforme a esos criterios. Cfr. A. Ruiz Miguel, «El principio de jerarquía normativa», en Revista Española de Derecho Constitucional, año 8, núm. 24, septiembre-diciembre, 1988. 
$\triangle$

DOXA-9 (1991) 